\title{
Mucinous Breast Carcinoma: Anatomo-Clinical Radiological and Therapeutic Features
}

\author{
Asma Korbi ${ }^{*}$, Houda Mhabrech ${ }^{2}$, Ennaceur Farouk ${ }^{1}$, Ons Cherif ${ }^{1}$, Amira Daldoul ${ }^{3}$, Chiraz Hafsa ${ }^{2}$, \\ Ahmed Hajji' ${ }^{1}$, Raja Faleh ${ }^{1}$
}

${ }^{1}$ Department of Gynecology and Obstetrics, University Hospital of Monastir, Monastir, Tunisia

${ }^{2}$ Department of Radiology B, University Hospital Fattouma Bourguiba of Monastir, Monastir, Tunisia

${ }^{3}$ Department of Oncology, University Hospital Fattouma Bourguiba of Monastir, Monastir, Tunisia

Email: *korbi.asma@live.fr

How to cite this paper: Korbi, A., Mhabrech, H., Farouk, E., Cherif, O., Daldoul, A., Hafsa, C., Hajji, A. and Faleh, R. (2018) Mucinous Breast Carcinoma: Anatomo-Clinical Radiological and Therapeutic Features. Open Access Library Journal, 5: e4858.

https://doi.org/10.4236/oalib.1104858

Received: August 21, 2018

Accepted: September 23, 2018

Published: September 26, 2018

Copyright (C 2018 by authors and Open Access Library Inc.

This work is licensed under the Creative Commons Attribution International License (CC BY 4.0).

http://creativecommons.org/licenses/by/4.0/

\begin{abstract}
Introduction: Mucinous carcinoma of the breast constitutes $1 \%$ to $4 \%$ of all breast cancers. Two different forms of presentation are distinguished: pure mucinous carcinoma and mixed mucinous carcinoma. The purpose of our work was to clarify the anatomo-clinical and radiological particularities of this rare form of breast cancer. Materials and methods: This was a retrospective study of a series of 14 cases of mucinous carcinoma of the breast, including 8 pure mucinous carcinomas and 6 mixed mucinous carcinomas treated at the Maternity and Neonatal Center of Monastir between January 2009 and December 2017. Results: The frequency of mucinous carcinoma of the breast in our study period was $1.9 \%$. The average of the patients was 55 years old. The average tumor size was $40 \mathrm{~mm}$. Mucinous carcinoma of the breast was pure in 8 cases, mixed in 6 cases. The clinical size of pure CM was smaller than that of mixed CM (41 mm vs $45 \mathrm{~mm})$ [p $=0.12]$. Axillary adenopathies were found in $28.5 \%$ of patients. Mammography detected a total of 19 masses with three cases of multifocality. The average size was $27 \mathrm{~mm}$, there was no significant difference between subtype sizes. Pure CMs had an oval shape in $83 \%$ of the cases with microlobulated contours in half of the cases, mixed CM rather had an irregular shape (71\%) with indistinct contours $(85 \%)(\mathrm{p}=0.01 / \mathrm{p}=$ 0.04). On ultrasound, pure CMs had a homogeneous hypoechogenic oval mass appearance with microlobulated contours enhancing ultrasound. The mixed CMs had an irregular shape of non-geometric contours and heterogeneous hypoechogenic appearance with posterior ultrasound attenuation $(\mathrm{p}=$ 0.06). Microcalcifications were present in $37 \%$ of pure $\mathrm{CM}$ and $60 \%$ of mixed $\mathrm{CM}(\mathrm{p}=0.13)$. Ganglionic invasion was noted in $28 \%$ of cases. The extension assessment was negative in all cases. The tumors were of low histological
\end{abstract}


grade with predominance of SBR I and II grades. They expressed hormone receptors in $85 \%$ of cases. Overexpression of the HER2 gene was noted in only one case. Conclusion: It is important to distinguish between the two pure and mixed forms, because the therapeutic attitude and the prognosis depend on it. The pure form remains a favorable prognosis, whereas that of the mixed form matches that of infiltrating ductal carcinomas.

\section{Subject Areas}

Oncology

\section{Keywords}

Mucinous Carcinoma, Pure, Mixed, Mammography, Ultrasound, MRI, Histological Grade

\section{Introduction}

Mucinous carcinoma or mucosal colloid (MC) is a rare histological form of mammary tumor [1]. It represents 1 to $4 \%$ of all breast cancers [2]. According to the World Health Organization (WHO), it is defined by the presence of extracellular mucus in which mucosecreting malignant tumor cells float [3]. Histologically, there are two types of MC: the pure MC and the mixed MC that associate foci of infiltrating ductal carcinoma next to the mucinous component. This distinction is crucial because of its prognostic value [3]. The positive diagnosis of these tumors is provided by echomammography and confirmed by ultrasound-guided microbiopsy or surgical excision. It is a mild cancer. Relapses are rare and usually late [4] [5]. Through a retrospective study of 14 cases of breast MC and a review of the literature, we will try to discuss the main features of this rare form of breast cancer.

\section{Materials and Methods}

This is a retrospective study of 14 patients with a breast $\mathrm{MC}$ including eight pure MC and six mixed MC, treated at Monastir Maternity and Neonatal Center over a period of 9 years from January 2009 to December 2017. We selected all patients with MC defined by the presence of extracellular mucin in which carcinomatous cells are bathed with or without the presence of non-specific ductal carcinoma component (CCnsp). Data was collected from patient records and transcribed on a pre-established computerized form. These data were relevant to demographic, anatomo-clinical and radiological characteristics.

\section{Results}

In our series we collected 14 patients with a breast MC from 728 patients diagnosed and treated for breast cancer at the Maternity and Neonatal Center of 
Monastir between January 2009 and December 2017. This rate represents $1.9 \%$ of cancers treated in the center during the same study period. The average age of our patients was 55 years (38 - 82 years). Only one patient in our series had a history of contra-lateral breast SCCs. A family history of breast cancer was noted in two patients (14\%). In $71.4 \%$ of the cases, the patients had consulted for appearance of a mammary mass, one had consulted for inflammatory breast and only one as part of a control balance (CCnsp of the contra-lateral breast). Both breasts were equally affected. The tumor was unique in 13 patients (92\%). A bifocal tumor was found in a patient with pure MC. The average clinical tumor size was $40 \mathrm{~mm}(20-80 \mathrm{~mm})$. Mucinous carcinoma of the breast was pure in 8 cases, mixed in 6 cases. Table 1 summarizes a comparison of clinical presentation of pure CM versus mixed CM.

Mammograms showed multifocal lesions in 3 cases. Pure CMs had an average size of $26 \mathrm{~mm}$ versus $29 \mathrm{~mm}$ for mixed CM without significant difference ( $\mathrm{p}=$ 0.17). They were $83 \%$ oval-shaped with microlobulated contours in half of the cases, while mixed CMs had an irregular shape in 57\% of cases with indistinct contours in $85 \%$ of cases, as shown in Figure 1 . The difference was significant ( $\mathrm{p}=$ $0.01 / \mathrm{p}=0.04)$. Microcalcifications were present in both subtypes $(\mathrm{p}=0.13)$.

On ultrasound, pure CMs had a homogeneous hypoechogenic oval mass appearance with microlobulated contours enhancing ultrasound. Mixed CMs had an irregular shape of non-geometric contours and heterogeneous hypoechogenic appearance with posterior attenuation of ultrasound without any notable difference as shown in Figure $2(\mathrm{p}=0.06)$.

Our patients were classified according to the 1986 TNM classification. Three

Table 1. Clinical presentation of pure CM versus mixed CM.

\begin{tabular}{|c|c|c|c|}
\hline & $\begin{array}{c}\text { PMBC } \\
\mathrm{N}=8\end{array}$ & $\begin{array}{c}\text { MMBC } \\
\mathrm{N}=6\end{array}$ & $p$ value \\
\hline Medianage & 59.5 & 49.5 & 0.09 \\
\hline Number of menopausal patients & $7(87 \%)$ & $2(33 \%)$ & 0.036 \\
\hline Personal history of breast cancer & - & 1 & - \\
\hline Family history of breast cancer & 1 & 1 & - \\
\hline Symptomatology: & & & 0.47 \\
\hline Palpable mass & $6(75 \%)$ & $4(66 \%)$ & - \\
\hline Inflammatorybreast & - & $1(16 \%)$ & - \\
\hline Localisation: & & & - \\
\hline Right breast & $4(50 \%)$ & $3(50 \%)$ & \\
\hline Left breast & $4(50 \%)$ & $3(50 \%)$ & \\
\hline Clinical size (mm) & 41 & 45 & 0.12 \\
\hline $\begin{array}{l}\text { Clinical limits } \\
\text { well limited } \\
\text { unclearly limited }\end{array}$ & $\begin{array}{c}8(100 \%) \\
0\end{array}$ & $\begin{array}{l}5(83 \%) \\
1(16 \%)\end{array}$ & 0.69 \\
\hline Lymphadenopathy & $2(25 \%)$ & $2(33 \%)$ & 0.42 \\
\hline
\end{tabular}



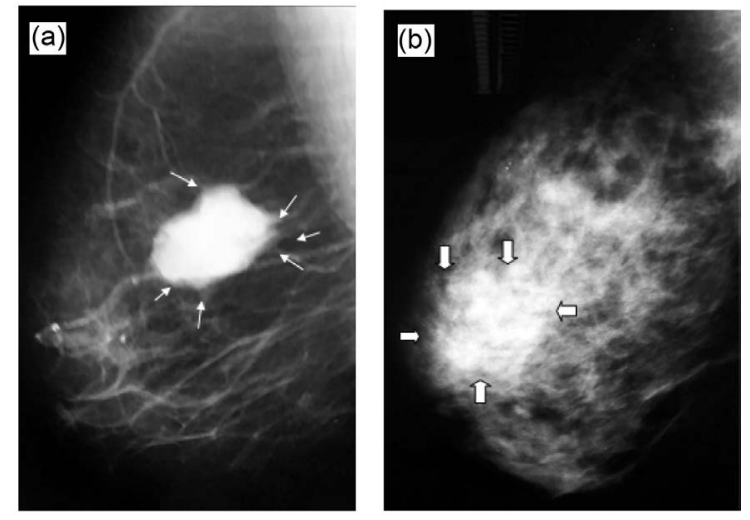

Figure 1. Mammography snapshot external oblique ((a), (b)): (a) Mass with microlobulated contours by location (arrows); (b) Retro-polar mass with indistinct contours (thick arrows).
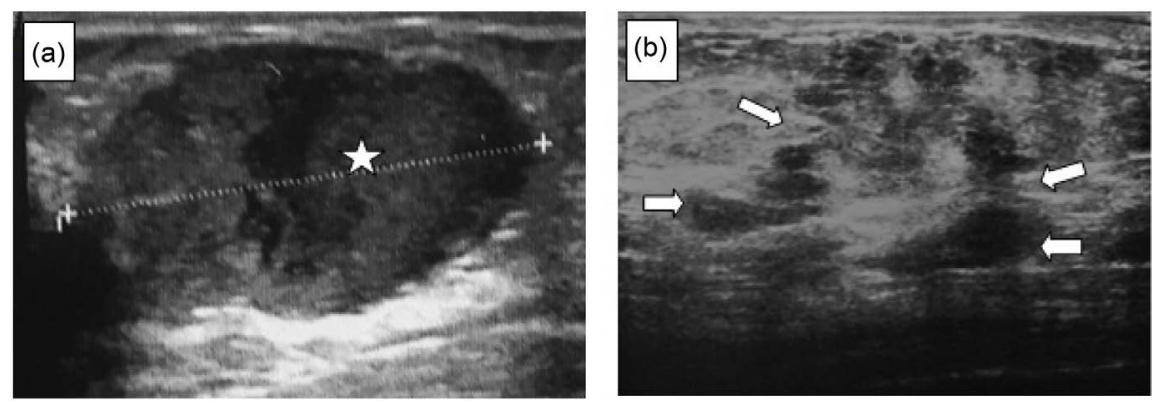

Figure 2. Mammary echography ((a), (b)): (a) Oval-shaped hematogenous mass (star); (b) Non-geometric hypoechoic mass (arrow).

(21\%) patients were classified as T1. Stage T2 represented a rate of $(28 \%)$. The T3 stage was found in three patients (14\%) and the $1 / 3$ of the patients (35\%) were classified T4. The radical surgery was performed on 13 patients. It consisted of a mastectomy with axillary dissection. Conservative surgery was performed in a case of pure MC. Pure MCs were classified as SBR I and SBR II in $50 \%$ of cases respectively. Mixed MCs were rated SBR I, SBR II and SBR III in equal ways. The average number of lymph nodes sampled was 12 (range 1 to 27). Ganglionic invasion was noted in 5 patients. Hormonal receptors were positive in 12 patients. HER2 was sought in all our patients. It was over-expressed at $(3+)$ in a single patient who had a pure $\mathrm{MC}$ and not expressed in the rest of the patients. Table 2 illustrates a comparison of histological presentation between pure $\mathrm{CM}$ versus mixed CM.

Chemotherapy was performed in 9 patients. This consisted of adjuvant chemotherapy in two patients (22\%) and neo-adjuvant chemotherapy in seven patients $(78 \%)$. Radiotherapy was performed in 10 patients (71.4\%). Hormone therapy was prescribed in 11 patients. Two patients developed metastases during the course of the progress. Overall survival at 5 years was $100 \%$ and survival recurrence free rate was $72 \%$ at 5 years. Overall survival for pure mucinous carcinoma is $74 \%$ while survival for mixed carcinoma is $59 \%$. 
Table 2. Histological presentation of pure CM versus mixed CM.

\begin{tabular}{cccc}
\hline & $\begin{array}{c}\text { PMBC } \\
(\mathbf{n}=12)\end{array}$ & $\begin{array}{c}\text { MMBC } \\
(\mathbf{n}=6)\end{array}$ & p value \\
\hline Microscopic limits: & $11(91 \%)$ & $1(17 \%)$ & $<0.01$ \\
Well limited & $1(9 \%)$ & $5(83 \%)$ & \\
unclearly limited & $27[10-60]$ & $42[17-90]$ & 0.12 \\
Tumoral size (cm) & & & \\
Histological grade: & $50 \%$ & $33 \%$ & 0.04 \\
SBRI & $50 \%$ & $33 \%$ & \\
SBRII & 0 & $33 \%$ & 0.17 \\
SBRIII & $25 \%$ & $50 \%$ & 0.86 \\
Invasive ganglion & & & \\
Hormonal receptors: & $87.5 \%$ & $84 \%$ & \\
Positive & $12.5 \%$ & $16 \%$ & 0.13 \\
Negative & & & \\
HER2 & 12.5 & 0 & \\
Positif & $87.5 \%$ & $100 \%$ & \\
Négatif & &
\end{tabular}

Note that the pure variant has a better survival compared to the mixed variant $(\mathrm{p}=0.045)$.

\section{Discussion}

MC is a particular histological form of breast carcinoma, first described in 1826 by Geschickter [6]. This is a rare entity that preferentially affects women over sixty years of age. It accounts for $7 \%$ of all malignant breast tumors after age 75 and $1 \%$ before age 35 [2] [7]. The average age at diagnosis is 65 years ( 48 to 82 years) [2] [3] [8]. In the literature, there were few studies that examined the influence of conventional breast cancer risk factors on MC. In terms of risk factors, no significant difference was found between MC, tubular carcinoma, and breast ITC [9]. Self-examination of a breast nodule was the most common telltale sign [10] [11] [12], and this was the case in our series. Bilateral character is rarely described in the literature, while multi-focality remains exceptional [5] [8] [9] [11]. Both mammography and ultrasound findings correlate with the histological type of the tumor and the extracellular mucin volume [13] [14] [15]. For pure MC it is a nodular mass circumscribed and multi-lobed with well-defined contours. The well-defined limits are correlated with the volume of extracellular mucin, so that they can be mistaken for benign formations [5] [16]. The mammographic aspect of mixed MC is nevertheless more suspect. It appears as a mass of irregular contours with ill-defined boundaries, or even speculated with the glandular tissue. The MRI aspect of the PMC is characteristic. Indeed, the signal intensity in $\mathrm{T} 1$ sequence after gadolinium injection varies according to the tumor concentration in extracellular mucin. In T2 sequence, the lesion is characterized by intense and homogenous contrast enhancement and dynamic 
analysis by a fine increase of the signal and then plateau, unlike CCI and mixed mucinous carcinoma which presents a heterogeneous enhancement with a Wash out appearance. However, the problem arises with phyllode tumors, medullary carcinomas, cysts, and abscesses that exhibit comparable behavior [14]-[19]. The interest of the extension assessment is not recommended by the majority of authors for the early stages [20] [21] [22]. However, the authors always recommend the extension assessment in patients with stage III breast cancer [22] [23]. In the literature, the majority of patients $(92 \%-97 \%)$ were classified as $\mathrm{T} 1$ or $\mathrm{T} 2$ according to the TNM classification [11] [12] [22] [24]. The breast MC is subdivided into two subtypes: Pure MC: characterized by the presence of tumor tissue completely surrounded by abundant extracellular mucus, with no infiltrating ductal component or when it is present, it does not exceed $10 \%$ of the overall tumor volume. The transition between mucus and surrounding connective tissue is abrupt. Mixed MC: characterized by the presence, in addition to the mucinous component, of an infiltrating ductal component. This constitutes more than $10 \%$ of the total tumor volume; the transition between extracellular mucus and adjacent carcinomatous tissue is progressive [3] [23]. The ER and positive PR rates reported in the literature in pure breast MC are $77 \%$ and $50 \%$, respectively, versus $74 \%$ and $52 \%$ for mixed MC [3]. In our series, hormone receptors were positive in 12 patients $(85 \%)$. For the surgical treatment, a radical treatment (a mastectomy with axillary dissection) is advisable on the other hand, there are consensually agreed indications concerning the conservative treatment such as unifocal invasive tumors, of size less than or equal to $3 \mathrm{~cm}$. distance from the nipple and without skin invasion [25] [26] [27]. Axillary dissection is an integral part of surgical treatment whether radical or conservative. The therapeutic value of cleaning is controversial. The ganglionic invasion in the pure MC of the breast is rare, it varies from 0 to $21 \%$ against $45 \%$ to $64 \%$ for the mixed $\mathrm{MC}$ and is relayed to the tumor size [9] [28]. Regarding chemotherapy, its indications in the breast MC are restricted [2] [9] [29] [30]. There is no consensus on protocols and number of courses. The data from the literature have identified some peculiarities specific to MC. The chemotherapy protocols are modeled on those used for other types of breast cancer. Trastuzumab has a place in the management of these tumors, in fact the efficacy of trastuzumab has been observed as an adjuvant, alone or in combination with concomitant or sequential chemotherapy, in patients with or without lymph node involvement. It improves overall survival, recurrence-free survival, and event time [31] [32] [33] [34]. Overall survival at 5 years ranges from $80 \%$ to $100 \%$ [2] [9] [11] [35]; at age 10 it ranges from $72.8 \%$ to $100 \%$ [1] [2] [11] [12]. Most authors have found better survival for MC compared with other infiltrating cancers. Recurrence-free survival at 5 years ranges from $81 \%$ to $91.6 \%$ [2] [9] [11]; at 10 years ranges from $75.3 \%$ to $90 \%$ [2] [12] [24]. For prognostic factors, the young age tumor size greater than $30 \mathrm{~mm}$, a tumor of high cellularity and ganglion invasion are factors of poor prognosis [9] [11] [24] [36]. However, no conclusions could be drawn 
about the impact of HER2 on prognosis [2] [9] [24].

\section{Conclusion}

Mucinous Carcinoma of the breast is a rare variety, occurring mainly after menopause. It is important to distinguish between the two pure and mixed forms, because the therapeutic attitude and the prognosis depend on it. The pure form remains a favorable prognosis, whereas that of the mixed form matches that of infiltrating ductal carcinomas.

\section{Conflicts of Interest}

The authors declare no conflicts of interest regarding the publication of this paper.

\section{References}

[1] Komaki, K., Sakamoto, G., Sugano, H., Morimoto, T. and Monden, Y. (1988) Mucinous Carcinoma of the Breast in Japan. A Prognosis Analysis Based on Morphologic Features. Cancer, 61, 989-996. https://doi.org/10.1002/1097-0142(19880301)61:5<989::AID-CNCR2820610522>3.0 .CO;2-E

[2] Thao, V., Yan, X., Funda, M.-B. and Nadeem, M. (2007) Long-Term Outcomes in Patients with Mucinous, Medllary, Tubular, and Invasive Ductual Carcinomas after Lumpectomy. American Journal of Surgery, 194, 527-531. https://doi.org/10.1016/j.amjsurg.2007.06.012

[3] Hartman, W.H., Ozzello, L., Sobin, L.H., et al. (1981) Histological Typing of Breast Tumors. 2nd Edition, World Health Organization, Geneva, 19-20.

[4] Fentiman, I.S., Millis, R.R., Smith, P., et al. (1997) Mucoid Breast Carcinomas: Histology and Prognosis. British Journal Of Cancer, 75, 1061-1065.

https://doi.org/10.1038/bjc.1997.180

[5] Geschickter, C.F. (1938) Gelatinous Mammary Cancer. Annals of Surgery, 108, 321-346. https://doi.org/10.1097/00000658-193809000-00001

[6] Fink, C., Lüdemann, H., Wasser, K. and Delorme, S. (2002) Incidental Finding of a Mucinous Carcinoma of the Breast by Dynamic MRI in a Patient with a History of Breast Trauma. Clinical Imaging, 26, 254-257. https://doi.org/10.1016/S0899-7071(02)00429-1

[7] Liu, H., Tan, H., Cheng, Y., Zhang, X., Gu, Y. and Peng, W. (2011) Imaging Findings in Mucinous Breast Carcinoma and Correlating Factors. European Journal of Radiology, 80, 706-712.

[8] Anan, K., Mitsuyama, S., Tamae, K., et al. (2001) Pathological Features of Mucinous Carcinoma of the Breast Are Favourable for Breast Conserving Therapy. European Journal of Surgical Oncology, 27, 459-463. https://doi.org/10.1053/ejso.2001.1142

[9] Kuhl, C.K. (2000) MRI of Breast Tumors. European Radiology, 10, 46-58. https://doi.org/10.1007/s003300050006

[10] Komenaka, I.K., El-Tamer, M.B., Troxel, A., Hamele-Bena, D., Joseph, L.A., et al. (2004) Pure Mucinous Carcinoma of the Breast. American Journal of Surgery, 187, 528-532. https://doi.org/10.1016/j.amjsurg.2003.12.039

[11] Chtourou, I., Krichen Makni, S., Bahri, I., et al. (2009) Carcinome colloïde pur du 
sein: étude anatmoclinique de sept cas. Cancer Radiothérapie, 13, 37-41. https://doi.org/10.1016/j.canrad.2008.06.004

[12] Memis, A., Ozdemir, N., Parildar, M., et al. (2000) Mucinous Colloïd Breast Cancer: Mammographic and US Features with Histologic Correlation. European Journal of Radiology, 35, 39-43. https://doi.org/10.1016/S0720-048X(99)00124-2

[13] Gilles, R. (2003) L'IRM mammaire. Techniques et valeur actuelle. Cancer Radiothérapie, 7, 115-119.

[14] Narissada, H., Aoki, T., Sasaguri, T., et al. (2006) Crrelation between Numeric Gadolinium-Enhanced Dynamic MRI Ratios and Prognostic Factors and Histologic Type of Breast Carcinoma. American Journal of Roentgenology, 187, 297-306. https://doi.org/10.2214/AJR.05.0696

[15] Diab, S.G., Gary, M.C., Kent Osborne, C., et al. (1999) Tumor Characteristics and Clinical Outcome of Tubular and Mucinous Breast Carcinomas. Journal of Clinical Oncology, 17, 1442-1452. https://doi.org/10.1200/JCO.1999.17.5.1442

[16] Kuwabara, M. (1991) MRI of Breast Tumors with Emphasis on Histopathologic Correlation. Nippon Acta Radiologica, 51, 1366-1374.

[17] Okafuji, T., Yabuuchi, H. and Hiroyasu, S. (2006) MR Imaging Features of Pure Mucinous Carcinoma of the Breast. European Journal of Radiology, 60, 405-413. https://doi.org/10.1016/j.ejrad.2006.08.006

[18] Kawashima, M., Tamakiy, Nonaka, T., et al. (2002) MR Imaging of Mucinous Carcinoma of the Breast. $A J R, 179,179-183$.

https://doi.org/10.2214/ajr.179.1.1790179

[19] Lam, W.W., Chu, W.C., Tse, G.M., Ma, T.K. and Tang, A.P. (2006) Role of Fine Needle Aspiration and Trucut Biopsy in Diagnosis of Mucinous Carcinoma of Breast from a Radiologist's Perspective. Clinical Imaging, 30, 6-10.

[20] Chen, E.A., Carlson, G.A., Coughlin, B.F., et al. (2000) Routine Chest Roentgenography in Unnecessary in the Work-Up for Stage I and II Breast Cancer. Journal of Clinical Oncology, 18, 3503-3506. https://doi.org/10.1200/JCO.2000.18.20.3503

[21] Ciatto, S., Pacini, P., Azzini, V., et al. (1998) Preoperative Staging of Primary Breast Cancer. A Multicentric Study. Cancer, 61, 1038-1040. https://doi.org/10.1002/1097-0142(19880301)61:5<1038::AID-CNCR2820610530>3. $\underline{0 . \mathrm{CO} ; 2-\mathrm{Z}}$

[22] Myers, R.E., Johnston, M., Pritchard, K., Levine, M. and Oliver, T. (2001) Breast Cancer Disease Site Group of the Cancer Care Ontario Practice Guidelines Initiative. Baseline Staging Tests in Primary Breast Cancer: A Practice Guideline. Canadian Medical Association Journal, 164, 1439-1444.

[23] Cox, M., Gilliand, R., OldingSmee, G.W., et al. (1992) An Evaluation of Radionuclide Scanning and Liver Ultrasonography for Staging Breast Cancer. Australian and New Zealand Journal of Surgery, 32, 550-555.

https://doi.org/10.1111/j.1445-2197.1992.tb07049.x

[24] Park, S., Koo, J., Kim, J.-H., et al. (2010) Clinicopathological Characteristics of Mucinous Carcinoma of the Breast in Korea: Comparison with Invasive Ductual Carcinoma-Not Otherwise Specified. Journal of Korean Medical Science, 25, 361-368. https://doi.org/10.3346/jkms.2010.25.3.361

[25] Barkley, C.R., Ligibel, J.A., Wong, J.S., et al. (2008) Mucinous Breast Carcinoma: A Large Contempory Series. The American Journal of Surgery, 196, 549-551. https://doi.org/10.1016/j.amjsurg.2008.06.013

[26] Fisher, B. and Anderson, S. (1994) Conservative Surgery for the Management of 
Invasive and Noninvasive Carcinoma of Breast: NSABP Trials. National Surgical Adjuvant Breast and Bowel Project. World Journal of Surgery, 18, 63-69. https://doi.org/10.1007/BF00348193

[27] Weber, B., Demange, L., Rigaud, C. and Fernandez-Valoni, A. (1998) Que resteil des indications de mastectomie totale pour cancer? Bulletin du Cancer, 85, 755-762.

[28] Andre, S., Cunha, F., Bernardo, M., et al. (1995) Mucinous Carcinoma of the Breast: A Pathologic Study of 82 Cases. Journal of Surgical Oncology, 58, 162-167. https://doi.org/10.1002/jso.2930580305

[29] Chu, J.-S. and Chang, K.-J. (1999) Mucin Expression in Mucinous Carcinoma and Other Invasive Carcinoma of the Breast. Cancer Letters, 142, 121-127. https://doi.org/10.1016/S0304-3835(99)00161-5

[30] Ishikawa, T., Hamaguchi, Y., Ichikawa, Y., et al. (2002) Locally Advanced Mucinous Carcinoma of the Breast with Sudden Growth Acceleration: A Case Report. Japanese Journal of Clinical Oncology, 32, 64-67. https://doi.org/10.1093/jjco/hyf012

[31] Zell, J.A., Tsang, W.Y., Taylor, T.H., Mehta, R.S. and Anton-Culver, H. (2009) Prognostic Impact of Human Epidermal Growth Factor-Like Receptor 2 and Hormone Receptor Status in Inflammatory Breast Cancer (IBC): Analysis of 2,014 IBC Patient Cases from the California Cancer Registry. Breast Cancer Research, 11, R9. https://doi.org/10.1186/bcr2225

[32] Ross, J.S., Slodkowska, E.A., Symmans, W.F., Pusztai, L., Ravdin, P.M., et al. (2009) The HER-2 Receptor and Breast Cancer: Ten Years of Targeted Anti-HER-2 Therapy and Personalized Medicine. Oncologist, 14, 320-368.

https://doi.org/10.1634/theoncologist.2008-0230

[33] Cobleigh, M.A., Vogel, C.L., Tripathy, D., Robert, N.J., Scholl, S., et al. (1999) Multinational Study of the Efficacy and Safety of Humanized Anti-HER2 Monoclonal Antibody in Women Who Have HER2-Overexpressing Metastatic Breast Cancer That Has Progressed after Chemotherapy for Metastatic Disease. Journal of Clinical Oncology, 17, 2639-2648. https://doi.org/10.1200/JCO.1999.17.9.2639

[34] Smith, I., Procter, M., Gelber, R.D., Guillaume, S., Feyereislova, A., et al. (2007) 2-Year Follow-Up of Trastuzumab after Adjuvant Chemotherapy in HER2-Positive Breast Cancer: A Randomised Controlled Trial. The Lancet, 369, 29-36. https://doi.org/10.1016/S0140-6736(07)60028-2

[35] Kurtz, J., Jacquemier, J., Torhorst, J., et al. (1989) Conservation Therapy for Breast Cancers Other than Infiltrating Ductal Carcinoma. Cancer, 63, 1630-1635. https://doi.org/10.1002/1097-0142(19890415)63:8<1630::AID-CNCR2820630833>3. $\underline{0 . \mathrm{CO} ; 2-\mathrm{U}}$

[36] Clayton, F. (1986) Pure Mucinous Carcinoma of the Breast: Morphologic Features and Prognostic Correlates. Human Pathology, 17, 34-38.

https://doi.org/10.1016/S0046-8177(86)80152-6 